\section{Long-term outcomes after small increases in serum creatinine during hospitalization}

Small increases in serum creatinine level have been associated with an increased risk of adverse short-term outcomes in hospitalized patients, but do such increases correlate with long-term risk of end-stage renal disease (ESRD) and death? To answer this question, Newsome et al. performed a retrospective study of elderly survivors of hospitalization for acute myocardial infarction (AMI).

Data from 87,094 African American or white patients aged 65 years or older (mean age 77.1 years) who had been admitted for AMI to non-federal US hospitals during the period February 1994 to July 1995 were analyzed. Follow-up data until June 2004 were examined. History of ESRD before hospitalization and use of acute hemodialysis during hospitalization were exclusion criteria. The median duration of follow-up was 4.1 years. The study found that all quartiles of increase in serum creatinine level during hospitalization were associated with increases in the risk of ESRD and the risk of death after hospital discharge. The adjusted hazard ratios for increases in serum creatinine level relative to a decrease or no change ranged from 1.45 for ESRD and 1.14 for death in the first quartile (increase of $9 \mu \mathrm{mol} / \mathrm{l}[0.1 \mathrm{mg} / \mathrm{dl}]$ ) to 3.26 and 1.39 , respectively, in the fourth quartile (increase of 53-265 $\mu \mathrm{mol} / \mathrm{l}[0.6-3.0 \mathrm{mg} / \mathrm{dl}]$ ).

The authors conclude that elderly survivors of AMI who exhibit changes in serum creatinine level during hospitalization should be closely monitored and aggressively treated.

Original article Newsome BB et al. (2008) Long-term risk of mortality and end-stage renal disease among the elderly after small increases in serum creatinine level during hospitalization for acute myocardial infarction. Arch Intern Med 168: 609-616

\section{Kidney dysfunction predicts sudden cardiac death in women with coronary heart disease}

Approximately $22 \%$ of all deaths in patients with end-stage renal disease are caused by sudden cardiac death (SCD), but it is not known whether less-severe kidney function is also associated with SCD. Deo et al. have established that moderate to severely impaired kidney function is an independent predictor of SCD in postmenopausal women with coronary heart disease.

This was a subanalysis of data from the randomized, placebo-controlled Heart and Estrogen/Progestin Replacement Study, which investigated the effect of daily medroxyprogesterone acetate and conjugated estrogens on the risk of cardiovascular events in postmenopausal women with a history of coronary heart disease. Baseline serum creatinine levels were available for 2,760 participants, of whom 230 had an estimated glomerular filtration rate (eGFR; as determined by the Modification of Diet in Renal Disease equation) of $<40 \mathrm{ml} / \mathrm{min}$, 1,503 an eGFR of $40-60 \mathrm{ml} / \mathrm{min}$, and 1,027 an eGFR of $>60 \mathrm{ml} / \mathrm{min}$.

During the follow-up period of 6.8 years, the rate of SCD was higher in women with moderate to advanced kidney dysfunction (eGFR $<40 \mathrm{ml} / \mathrm{min}$ ) than in those with less severely impaired renal function (eGFR 40-60 $\mathrm{ml} / \mathrm{min}$ and $>60 \mathrm{ml} / \mathrm{min} ; 1.7 \%, 0.6 \%$ and $0.5 \%$ per year, respectively; $P<0.001$ for trend). There was no significant difference in the incidence of SCD between women with an eGFR of $>60 \mathrm{ml} / \mathrm{min}$ and those with an eGFR of $40-60 \mathrm{ml} / \mathrm{min}$. Adjustment for incident myocardial infarction and congestive heart failure-both of which were predictors of SCD-only slightly attenuated the association between kidney dysfunction and SCD $(P<0.003)$.

Original article Deo R et al. (2008) Kidney dysfunction and sudden cardiac death among women with coronary heart disease. Hypertension 51: 1578-1582

\section{Benefit from blood-pressure- lowering drugs is linked to urinary albumin level}

In an observational analysis of Dutch adults who participated in the community-based PREVEND trial, Boersma et al. have shown that hypertensive people with elevated urinary albumin levels receive a greater benefit from blood-pressure-lowering drugs, especially those that interfere with the renin-angiotensin system, than do individuals with normal urinary albumin levels.

The analysis included data from 1,185 individuals aged $28-75$ years who at baseline had elevated blood pressure $(\geq 140 / 90 \mathrm{mmHg})$, were not taking blood-pressure-lowering agents and 